\title{
Identification of 45 novel SNPs in the 83-kb region containing peptidylarginine deiminase types 1 and 3 loci on chromosomal band 1p36.13
}

Received: 23 March 2004/ Accepted: 25 March 2004/Published online: 19 May 2004

(C) The Japan Society of Human Genetics and Springer-Verlag 2004

\begin{abstract}
Peptidylarginine deiminases (PADIs) are posttranslational modification enzymes that catalyze the conversion of protein-bound arginine residues into citrulline residues in the presence of calcium ions. Among PADIs, PADI4 was identified as a rheumatoid arthritissusceptibility gene (Suzuki et al. in Nat Genet 34:395, 2003). We identified a total of 87 single nucleotide polymorphisms (SNPs) in PADII and PADI3gene loci. Following a comparison of our data with SNPs in the dbSNP database in the National Center for Biotechnology Information, 45 SNPs are considered to be novel: 33 were identified in the $P A D I I$ gene locus and 12 in the $P A D I 3$ gene locus. We also identified two insertiondeletion polymorphisms in introns of the PADI1. The high-resolution map that we constructed in this study will serve as a useful resource for analyzing gene scans of complex diseases mapped to this local segment on chromosomal band 1p36.13.
\end{abstract}

Keywords Single nucleotide polymorphism (SNP) · Japanese population · High-density SNP map ·

Rheumatoid arthritis $\cdot$ Peptidylarginine deiminase 1 gene $\cdot$ Peptidylarginine deiminase 3 gene

\section{Introduction}

Peptidylarginine deiminases (PADIs) are post-translational modification enzymes that catalyze the conversion of protein-bound arginine residues into citrulline residues in the presence of calcium ions. To date, four human PADI genes, type 1 to type 4, have been isolated and characterized by cDNA sequences, expression patterns, and biochemical analyses (Nakashima et al. 1999; Kanno et al. 2000; Ishigami et al. 2002; Guerrin et al. 2003). Three of four genes, PADI1, PADI3, and PADI4, lie within an approximately $160-\mathrm{kb}$ region on chromosome $1 \mathrm{p} 36.13$. PADI2 is located at $86-\mathrm{kb}$ distal to the $P A D I 1,3,4$ gene cluster.

We previously reported one of four $P A D I$ genes, $P A D I 4$, to be a rheumatoid arthritis-susceptibility gene by a case-control association study using single nucleotide polymorphisms (SNPs) and subsequent functional assays (Suzuki et al. 2003). In the course of this association study, we constructed fine-scale SNP maps of the $P A D I$ gene cluster region by a direct sequencing method and the SNP information derived from the JSNP database (Haga et al. 2002). In this report, we describe more detailed information of genetic variations in the $83-\mathrm{kb}$ region corresponding to the PADII/PADI3 loci, including 45 novel SNPs and two insertion-deletion polymorphisms in the Japanese population.

\section{Subjects and methods}

Laboratory for Pharmacogenetics,

Research Group of Personalized Medicine,

RIKEN SNP Research Center,

c/o RIKEN Yokohama Institute,

1-7-22 Suehiro-cho, Tsurumi, Yokohama,

Kanagawa 230-0045, Japan

E-mail: iidaari@src.riken.go.jp

Tel.: + 81-45-5039599

Fax: + 81-45-5039326

Y. Nakamura

Laboratory of Molecular Medicine,

Human Genome Center, Institute of Medical Science,

The University of Tokyo, Tokyo, Japan
Samples of peripheral blood were obtained with written informed consent form 48 healthy Japanese volunteers for this study. Polymerase chain reaction (PCR) experiments and DNA sequencing were performed according to the methods described previously (Iida et al. 2003). In brief, on the basis of the genomic sequence from the Genbank database (accession number AL590644.13), we designed primer sites to amplify each gene locus in its entirely, excluding most of the regions that corre- 


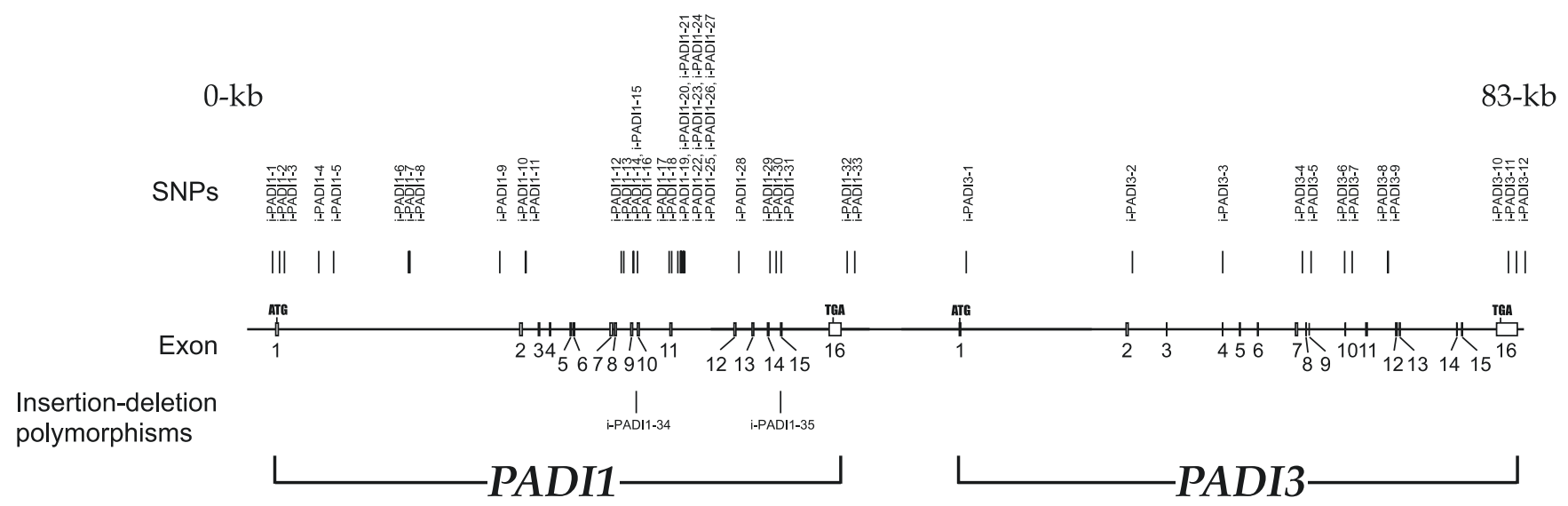

Fig. 1 A fine-scale single nucleotide polymorphism (SNP) map in the $83-\mathrm{kb}$ region including the $P A D I 1$ and $P A D I 3$. Exons and introns are represented by rectangles and horizontal lines, respectively. SNPs are indicated above the lines (designations correspond to the left-most column of Table 1). Two insertion-deletion polymorphisms are indicated below the map

sponded to human repetitive sequences predicted by the RepeatMasker program (http://www.repeatmasker. genome.washington.edu/cgi-bin/RepeatMasker). PCR was performed using $20 \mathrm{ng}$ of a mixture of genomic DNAs from three individuals. All 16 mixed samples were amplified in the GeneAmp PCR system 9700 (PE Applied Biosystems, Foster City, CA, USA) under the following conditions: initial denaturation at $94^{\circ} \mathrm{C}$ for $2 \mathrm{~min}$, followed by 35 cycles of denaturation at $94^{\circ} \mathrm{C}$ for $30 \mathrm{~s}$, annealing at $60^{\circ} \mathrm{C}$ for $30 \mathrm{~s}$, extension at $72^{\circ} \mathrm{C}$ for $2 \mathrm{~min}$, and postextension at $72^{\circ} \mathrm{C}$ for $7 \mathrm{~min}$. Products obtained from the PCR experiments served as templates for direct sequencing and detection of SNPs using the fluorescent dye-terminator Cycle-Sequencing method. All SNPs detected by the Polyphred computer program (Nickerson et al. 1997) were confirmed by sequencing both strands of each PCR product.

\section{Results and discussion}

By direct sequencing of DNA from 48 Japanese individuals, we explored SNPs in an $83-\mathrm{kb}$ genomic region including the PADI1/PADI3 loci except most of the regions containing human repetitive sequences. The ex- tent of each screened genomic sequence was $16.8-\mathrm{kb}$ for the PADII locus and 13.1-kb for the PADI3 locus. Consequently, we identified a total of 87 SNPs from the regions (SNPs were distributed every 344 nucleotides on average). By comparing our data with the SNPs deposited in the dbSNP database in the National Center for Biotechnology Information, USA, we considered 45 of these SNPs to be novel as of beginning of March 2004; 33 were identified in the PADII locus and 12 in the PADI3 locus (Fig. 1). Detailed information for these genetic variations is given in Table 1. Subregional distributions of novel SNPs were as follows: one in $5^{\prime}$ flanking regions, four in coding regions, 35 in introns, two in $3^{\prime}$ untranslated regions, and three in $3^{\prime}$ flanking regions. Of four SNPs identified in the coding regions, three were nonsynonymous substitutions: 1289T $>$ A (Ile430Asn) in exon 11 and $1652 \mathrm{G}>\mathrm{A}$ (Arg551His) in exon 15 of the PADI1, and $380 \mathrm{G}>\mathrm{A}$ (Gly127Glu) in exon 4 of the $P A D I 3$. All three could affect structures and/or biological functions of the respective gene products. In addition, the overall frequencies of nucleotide substitutions were counted as $31 \%$ for $A / G, 33 \%$ for $\mathrm{C} / \mathrm{T}, 7 \%$ for $\mathrm{A} / \mathrm{C}, 11 \%$ for $\mathrm{C} / \mathrm{G}, 11 \%$ for $\mathrm{G} / \mathrm{T}$, and $7 \%$ for $\mathrm{T} / \mathrm{A}$. The transitions occurred 1.8 times more frequently than transversions. We also found two insertion-deletion polymorphisms in the introns of the $P A D I 1$.

The SNP map that we constructed in this study will serve as a useful resource for analyzing gene scans of complex diseases mapped to this local segment on chromosomal band 1p36.13. We hope that the genetic variations can contribute to further investigations for designing personalized medical care. 
Table 1 Characterization of 45 novel SNPs and two insertion-deletion polymorphisms in the PADII and PADI3gene loci. $d b S N P$ database of single nucleotide polymorphisms, del deletion polymorphsim

\begin{tabular}{|c|c|c|c|c|c|c|c|}
\hline I.D. & Location & Position $^{\mathrm{a}}$ & $5^{\prime}$ Flanking sequence ${ }^{b}$ & Variation $^{\mathrm{c}}$ & $3^{\prime}$ Flanking sequence ${ }^{b}$ & Substitution & Repetitive \\
\hline \multicolumn{8}{|c|}{ Peptidylarginine deiminase type 1} \\
\hline i-PADI1-1 & $\begin{array}{l}5^{\prime} \text { Flanking } \\
\text { region }\end{array}$ & -194 & gcaggtgagtcctgcttcgc & $\mathrm{C} / \mathrm{T}$ & cggggcccccagttgaagct & & \\
\hline i-PADI1-2 & Intron 1 & 117 & aggatgctggcaggagatag & $\mathrm{C} / \mathrm{A}$ & agagaaggtggagttggctg & & \\
\hline i-PADI1-3 & Intron 1 & 432 & agggacgtgtgtggtagctt & $\mathrm{G} / \mathrm{T}$ & accettggaggtcagccaga & & \\
\hline i-PADI1-4 & Intron 1 & 2,838 & tactggtgggaggggggaag & $\mathrm{C} / \mathrm{A}$ & ccccagaagaggaggtgttg & & \\
\hline i-PADI1-5 & Intron 1 & 3,916 & ttacaggattgacactgaca & $\mathrm{C} / \mathrm{G}$ & taatttccagcgaccgacag & & \\
\hline i-PADI1-6 & Intron 1 & 9,170 & gtgactcctgtagtggtaat & $\mathrm{G} / \mathrm{C}$ & atttggcettttttatttgg & & \\
\hline i-PADI1-7 & Intron 1 & 9,223 & aaatctgtgacctcacctga & $\mathrm{C} / \mathrm{A}$ & tgtgcatttggggacatgcc & & \\
\hline i-PADI1-8 & Intron 1 & 9,244 & tgtgcatttggggacatgcc & $\mathrm{A} / \mathrm{G}$ & gtactgcatatctgggggtt & & \\
\hline i-PADI1-9 & Intron 1 & 15,573 & gccetgttggttttgctctg & $\mathrm{C} / \mathrm{G}$ & gtgccetgtagcctgagttt & & \\
\hline i-PADI1-10 & Intron 2 & 215 & ccagtatcacacatgtacac & $\mathrm{G} / \mathrm{A}$ & tgagtactcccacaaacac & & \\
\hline i-PADI1-11 & Intron 2 & 277 & cagattcacagacaacttca & $\mathrm{C} / \mathrm{T}$ & attaatccetgtattcagcc & & \\
\hline i-PADI1-12 & Intron 8 & 385 & ccttgcetgctatggacatg & $\mathrm{G} / \mathrm{A}$ & gaacccagtctctgcaagcc & & \\
\hline i-PADI1-13 & Intron 8 & 541 & gaccactggaaaaagccaca & $\mathrm{G} / \mathrm{T}$ & ttgagctggtcataagttaa & & \\
\hline i-PADI1-14 & Intron 9 & 51 & cagcctggettggggaaagg & $\mathrm{G} / \mathrm{A}$ & aagccgcacaggtgectgat & & \\
\hline i-PADI1-15 & Intron 9 & 102 & tcgctagagaagccacggca & $\mathrm{G} / \mathrm{A}$ & cctgggccaggcctctgggg & & \\
\hline i-PADI1-16 & Intron 9 & 336 & aaccacccgaaaaggtcac & $\mathrm{T} / \mathrm{C}$ & gtggetttttcatctctcce & & \\
\hline i-PADI1-17 & Intron 10 & 2,098 & cetcgtcctgtcetcactga & $\mathrm{C} / \mathrm{T}$ & ctctccttcttggggtgggg & & \\
\hline i-PADI1-18 & Coding region 11 & 1,289 & ggaatacccetgggecgga & $\mathrm{T} / \mathrm{A}$ & cctcatcgggagcagcttcc & Ile430Asn & \\
\hline i-PADI1-19 & Intron 11 & 442 & ttaaaagggtcctgaggtca & $\mathrm{T} / \mathrm{C}$ & gaagttcccagggcaggtgg & & \\
\hline i-PADI1-20 & Intron 11 & 590 & aacgctcaaacaggtggcga & $\mathrm{T} / \mathrm{C}$ & gtctgttaggtcctgctgag & & \\
\hline i-PADI1-21 & Intron 11 & 647 & ctgcaggtaaccaaagaatg & $\mathrm{A} / \mathrm{T}$ & $\begin{array}{l}(\mathrm{C} / \mathrm{G}) \text { gtgtgcagt }(\mathrm{T} / \mathrm{C}) \\
\text { cagggtcct }\end{array}$ & & \\
\hline i-PADI1-22 & Intron 11 & 648 & $\begin{array}{l}\text { tgcaggtaaccaaagaatg } \\
(\mathrm{A} / \mathrm{T})\end{array}$ & $\mathrm{C} / \mathrm{G}$ & gtgtgcagt( $\mathrm{T} / \mathrm{C})$ cagggtcctg & & \\
\hline i-PADI1-23 & Intron 11 & 658 & $\begin{array}{l}\text { caaagaatg }(\mathrm{A} / \mathrm{T}) \\
(\mathrm{C} / \mathrm{G}) \operatorname{gtgtgcagt}\end{array}$ & $\mathrm{T} / \mathrm{C}$ & cagggtcctggtgggtcccc & & \\
\hline i-PADI1-24 & Intron 11 & 730 & aagccetggagatcaccttc & $\mathrm{C} / \mathrm{T}$ & tgtttgtggggtttttgcat & & \\
\hline i-PADI1-25 & Intron 11 & 797 & aagccetggetgaccatctg & $\mathrm{G} / \mathrm{A}$ & tctccagcccagaggagtca & & \\
\hline i-PADI1-26 & Intron 11 & 840 & cagtgctgagaaggtcacag & $\mathrm{C} / \mathrm{T}$ & ctcacctggtgaatctggca & & \\
\hline i-PADI1-27 & Intron 11 & 915 & cetggcacctacccaggcac & $\mathrm{G} / \mathrm{A}$ & taactgggtgcccagacagg & & \\
\hline i-PADI1-28 & Intron 12 & 229 & aggaatgatattgataataa & $\mathrm{T} / \mathrm{C}$ & tggtaatatttattgagcac & & + \\
\hline i-PADI1-29 & Intron 14 & 91 & ggttgggtcagagattcagc & $\mathrm{G} / \mathrm{A}$ & ctggagctctcatggtcctg & & \\
\hline i-PADI1-30 & Intron 14 & 503 & ggcaggagggecettacccc & $\mathrm{T} / \mathrm{G}$ & tcccggtagtgeccagcget & & \\
\hline i-PADI1-31 & Coding region 15 & 1,652 & gaaatgcattgactggaacc & $\mathrm{G} / \mathrm{A}$ & taatgtgctgaagcgggagc & Arg551His & \\
\hline i-PADI1-32 & $\begin{array}{l}\text { 3' Flanking } \\
\text { region }\end{array}$ & 410 & gcaggcagtgggaggggaag & $\mathrm{G} / \mathrm{T}$ & cttgcceggtctctctcagc & & \\
\hline i-PADI1-33 & $\begin{array}{l}\text { 3' Flanking } \\
\text { region }\end{array}$ & 974 & ctgagaaagagctgtgggta & $\mathrm{G} / \mathrm{A}$ & gaagggagtgagcattgcag & & \\
\hline i-PADI1-34 & Intron 9 & 245 & cetcacttctacctctgcce & $\mathrm{C} / \mathrm{del}$ & tcaccaggtcttagggaaga & & \\
\hline i-PADI1-35 & Intron 14 & $815-824$ & cttggccagtgttatgctct & $\begin{array}{l}\text { TCCTAGTCCT/ } \\
\text { del }\end{array}$ & gggctgcccagctgcetcct & & \\
\hline \multicolumn{8}{|c|}{ Peptidylarginine deiminase type 3} \\
\hline i-PADI3-1 & Intron 1 & 324 & gctgcaggttgggctgagga & $\mathrm{T} / \mathrm{C}$ & ctgtctggggcacacaagaa & & \\
\hline i-PADI3-2 & Intron 2 & 254 & gggataatcataaccctgt & $\mathrm{C} / \mathrm{T}$ & tcctatcattgctgtggagt & & + \\
\hline i-PADI3-3 & Coding region 4 & 380 & ttgcgacctgaactgtgagg & $\mathrm{G} / \mathrm{A}$ & aaggcaggacaggaactttg & Gly127Glu & \\
\hline i-PADI3-4 & Intron 7 & 275 & aataaagggcagtggggagg & $\mathrm{A} / \mathrm{G}$ & ctccattcctggtaagtcta & & \\
\hline i-PADI3-5 & Intron 9 & 71 & gaggcettcatttgggggec & $\mathrm{A} / \mathrm{G}$ & ccgagaaacatccaggagga & & \\
\hline i-PADI3-6 & Coding region 10 & 1,080 & ctgggctacgttcaggcgcc & $\mathrm{G} / \mathrm{T}$ & cacaagaccetcccggtggt & Pro360Pro & \\
\hline i-PADI3-7 & Intron 10 & 375 & gggtcagaccaaccctgttc & $\mathrm{C} / \mathrm{T}$ & gttacttacatgctgtgtga & & + \\
\hline i-PADI3-8 & Intron 11 & 1,261 & agtggaattttaaaatatac & $\mathrm{G} / \mathrm{A}$ & tatctggctgggtgcagtgg & & \\
\hline i-PADI3-9 & Intron 11 & 1,326 & tgtgaggctgaggcgggcag & $\mathrm{A} / \mathrm{T}$ & tcacttgaggtcaggagttc & & + \\
\hline i-PADI3-10 & $3^{\prime}$ UTR & 2,571 & cccagatgttgaccetcacc & $\mathrm{C} / \mathrm{T}$ & agcgtgagctgtcacatagt & & \\
\hline i-PADI3-11 & 3' UTR & 3,082 & cgaggtgtgtgccagctaca & $\mathrm{C} / \mathrm{T}$ & gtgtgttctgtatgggtcca & & \\
\hline i-PADI3-12 & $\begin{array}{l}3^{\prime} \text { Flanking } \\
\text { region }\end{array}$ & 484 & cagaaacacactcaagctca & $\mathrm{C} / \mathrm{G}$ & cccgggetgggtggetagag & & \\
\hline
\end{tabular}

\footnotetext{
${ }^{a}$ Nucleotide numbering is according to the mutation nomenclature (den Dunnen and Antonarakis 2000)

${ }^{b}$ Both $5^{\prime}$ and $3^{\prime}$ flanking sequences to each variation are denoted by small letters

${ }^{c}$ Variation is shown by capital letter
} 


\section{References}

den Dunnen JT, Antonarakis SE (2000) Mutation nomenclature extensions and suggestions to describe complex mutations: a discussion. Hum Mutat 15:7-12

Guerrin M, Ishigami A, Mechin MC, Nachat R, Valmary S, Sebbag M, Simon M, Senshu T, Serre G (2003) cDNA cloning, gene organization and expression analysis of human peptidylarginine deiminase type I. Biochemistry 370:167-174

Haga H, Yamada R, Ohnishi Y, Nakamura Y, Tanaka T (2002) Gene-based SNP discovery as part of the Japanese Millennium Genome Project: identification of 190,562 genetic variations in the human genome. Single-nucleotide polymorphism. J Hum Genet 47:605-610

Iida A, Saito S, Sekine A, Mishima C, Kitamura Y, Kondo K, Harigae S, Osawa S, Nakamura Y (2003) Catalog of 668 SNPs detected among 31 genes encoding potential drug targets on the cell surface. J Hum Genet 48:23-46

Ishigami A, Ohsawa $\mathrm{T}$, Asaga $\mathrm{H}$, Akiyama $\mathrm{K}$, Kuramoto $\mathrm{M}$, Maruyama N (2002) Human peptidylarginine deiminase type II: molecular cloning, gene organization, and expression in human skin. Arch Biochem Biophys 407:25-31
Kanno T, Kawada A, Yamanouchi J, Yosida-Noro C, Yoshiki A, Shiraiwa M, Kusakabe M, Manabe M, Tezuka T, Takahara H (2000) Human peptidylarginine deiminase type III: molecular cloning and nucleotide sequence of the cDNA, properties of the recombinant enzyme, and immunohistochemical localization in human skin. J Invest Dermatol 115:813-823

Nakashima K, Hagiwara T, Ishigami A, Nagata S, Asaga H, Kuramoto M, Senshu T, Yamada M (1999) Molecular characterization of peptidylarginine deiminase in HL-60 cells induced by retinoic acid and lalpha,25-dihydroxyvitamin $\mathrm{D}(3)$. J Biol Chem 274:27786-27792

Nickerson DA, Tobe VO, Taylor SL (1997) PolyPhred: automating the detection and genotyping of single nucleotide substitutions using fluorescence-based resequencing. Nucleic Acids Res 25:2745-2751

Suzuki A, Yamada R, Chang X, Tokuhiro S, Sawada T, Suzuki M, Nagasaki M, Nakayama-Hamada M, Kawaida R, Ono M, Ohtsuki M, Furukawa H, Yoshino S, Yukioka M, Tohma S, Matsubara T, Wakitani S, Teshima R, Nishioka Y, Sekine A, Iida A, Takahashi A, Tsunoda T, Nakamura Y, Yamamoto K (2003) Functional haplotypes of PADI4, encoding citrullinating enzyme peptidylarginine deiminase 4 , are associated with rheumatoid arthritis. Nat Genet 34:395-402 